University of Nebraska - Lincoln

DigitalCommons@University of Nebraska - Lincoln

2008

\title{
A Survey on Spectrum Management in Cognitive Radio Networks
}

Ian F. Akyildiz

Georgia Institute of Technology

Won-Yeol Lee

Georgia Institute of Technology

Mehmet C. Vuran

Georgia Institute of Technology, mcvuran@cse.unl.edu

Shantidev Mohanty

Georgia Institute of Technology

Follow this and additional works at: https://digitalcommons.unl.edu/csearticles

Part of the Computer Sciences Commons

Akyildiz, Ian F.; Lee, Won-Yeol; Vuran, Mehmet C.; and Mohanty, Shantidev, "A Survey on Spectrum Management in Cognitive Radio Networks" (2008). CSE Journal Articles. 87.

https://digitalcommons.unl.edu/csearticles/87

This Article is brought to you for free and open access by the Computer Science and Engineering, Department of at DigitalCommons@University of Nebraska - Lincoln. It has been accepted for inclusion in CSE Journal Articles by an authorized administrator of DigitalCommons@University of Nebraska - Lincoln. 


\section{A Survey on Spectrum Management in Cognitive Radio Networks}

Ian F. Akyildiz, Won-Yeol Lee, Mehmet C. Vuran, and Shantidev Mohanty, Georgia Institute of Technology

\begin{abstract}
Cognitive radio networks will provide high bandwidth to mobile users via heterogeneous wireless architectures and dynamic spectrum access techniques. However, CR networks impose challenges due to the fluctuating nature of the available spectrum, as well as the diverse QoS requirements of various applications. Spectrum management functions can address these challenges for the realization of this new network paradigm. To provide a better understanding of CR networks, this article presents recent developments and open research issues in spectrum management in CR networks. More specifically, the discussion is focused on the development of CR networks that require no modification of existing networks. First, a brief overview of cognitive radio and the $\mathrm{CR}$ network architecture is provided. Then four main challenges of spectrum management are discussed: spectrum sensing, spectrum decision, spectrum sharing, and spectrum mobility.
\end{abstract}

\section{INTRODUCTION}

Current wireless networks are characterized by a static spectrum allocation policy, where governmental agencies assign wireless spectrum to license holders on a long-term basis for large geographical regions. Recently, because of the increase in spectrum demand, this policy faces spectrum scarcity in particular spectrum bands. In contrast, a large portion of the assigned spectrum is used sporadically, leading to underutilization of a significant amount of spectrum [1]. Hence, dynamic spectrum access techniques were recently proposed to solve these spectrum inefficiency problems.

The key enabling technology of dynamic spectrum access techniques is cognitive radio (CR) technology, which provides the capability to share the wireless channel with licensed users in an opportunistic manner. CR networks are envisioned to provide high bandwidth to mobile users via heterogeneous wireless architectures and dynamic spectrum access techniques. This goal can be realized only through dynamic and efficient spectrum management techniques. CR networks, however, impose unique challenges due to the high fluctuation in the available spectrum, as well as the diverse quality of service (QoS) requirements of various applications.

In order to address these challenges, each $\mathrm{CR}$ user in the CR network must:

- Determine which portions of the spectrum are available

- Select the best available channel

- Coordinate access to this channel with other users

- Vacate the channel when a licensed user is detected [2]

These capabilities can be realized through spectrum management functions that address four main challenges: spectrum sensing, spectrum decision, spectrum sharing, and spectrum mobility.

This article presents a definition, the functions, and the current research challenges of spectrum management in CR networks. More specifically, we focus our discussion on the development of CR networks that require no modification in existing networks. An overview of CR technology is provided, and the CR network architecture is presented. We explain the concept of spectrum management and the required functionalities. Then we describe spectrum sensing, spectrum decision, spectrum sharing, and spectrum mobility concepts.

\section{Cognitive Radio Technology}

The key enabling technologies of CR networks are the cognitive radio techniques that provide the capability to share the spectrum in an opportunistic manner. Formally, a CR is defined as a radio that can change its transmitter parameters based on interaction with its environment [1]. From this definition, two main characteristics of cognitive radio can be defined [3]:

- Cognitive capability: Through real-time interaction with the radio environment, the portions of the spectrum that are unused at a specific time or location can be identified. As shown in Fig. 1a, CR enables the usage of temporally unused spectrum, referred to as spectrum hole or white space. Consequently, the best spectrum can be selected, shared with other users, and exploited with- 


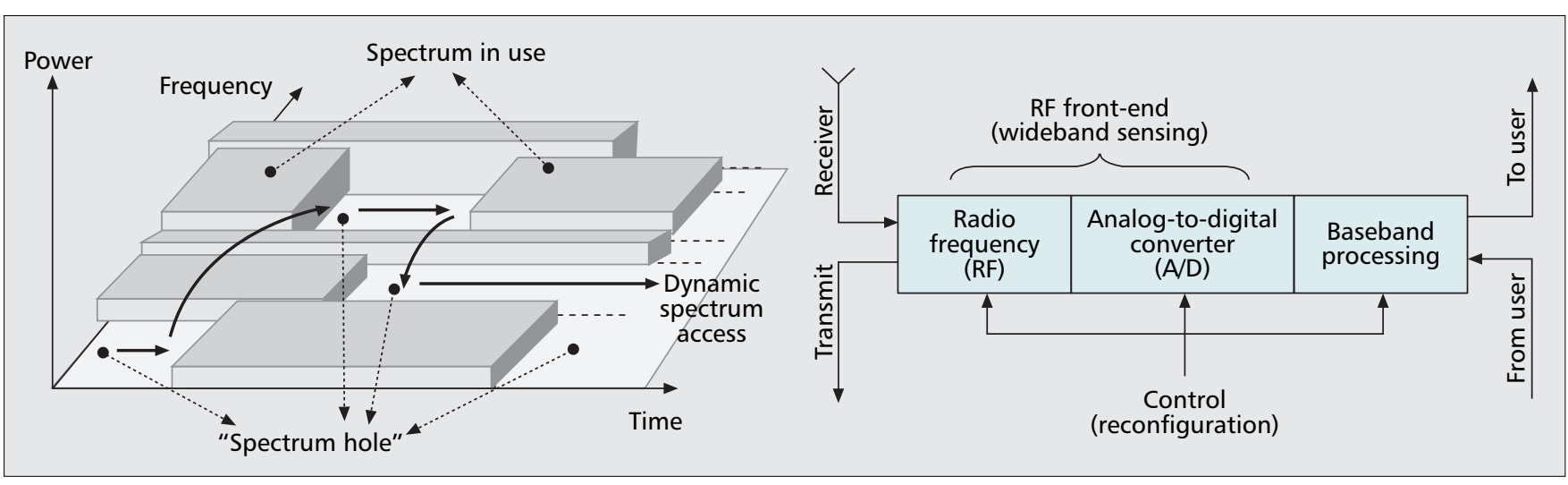

Figure 1. Overview of cognitive radio: a) the spectrum hole concept; b) cognitive radio transceiver architecture.

out interference with the licensed user.

- Reconfigurability: A CR can be programmed to transmit and receive on a variety of frequencies, and use different access technologies supported by its hardware design [4]. Through this capability, the best spectrum band and the most appropriate operating parameters can be selected and reconfigured.

In order to provide these capabilities, CR requires a novel radio frequency (RF) transceiver architecture. The main components of a CR transceiver are the radio front-end and the baseband processing unit that were originally proposed for software-defined radio (SDR), as shown in Fig. 1b [4]. In the RF front-end the received signal is amplified, mixed, and analogto-digital (A/D) converted. In the baseband processing unit, the signal is modulated/ demodulated. Each component can be reconfigured via a control bus to adapt to the time-varying RF environment. The novel characteristic of the CR transceiver is the wideband RF front-end that is capable of simultaneous sensing over a wide frequency range. This functionality is related mainly to the RF hardware technologies, such as wideband antenna, power amplifier, and adaptive filter. RF hardware for the CR should be capable of being tuned to any part of a large range of spectrum. However, because the CR transceiver receives signals from various transmitters operating at different power levels, bandwidths, and locations; the RF front-end should have the capability to detect a weak signal in a large dynamic range, which is a major challenge in CR transceiver design [5].

\section{Cognitive Radio NetWork ARCHITECTURE}

A comprehensive description of the CR network architecture is essential for the development of communication protocols that address the dynamic spectrum challenges. The CR network architecture is presented in this section.

\section{Network Components}

The components of the CR network architecture, as shown in Fig. 2, can be classified as two groups: the primary network and the CR network. The primary network (or licensed network) is referred to as an existing network, where the primary users have a license to operate in a certain spectrum band. If primary networks have an infrastructure, primary user activities are controlled through primary base stations. Due to their priority in spectrum access, the operations of primary users should not be affected by unlicensed users.

The CR network (also called the dynamic spectrum access network, secondary network, or unlicensed network) does not have a license to operate in a desired band. Hence, additional functionality is required for $C R$ users to share the licensed spectrum band. CR networks also can be equipped with $C R$ base stations that provide single-hop connection to CR users. Finally, CR networks may include spectrum brokers that play a role in distributing the spectrum resources among different CR networks [6].

\section{SPECTRUM HeterogeneITY}

CR users are capable of accessing both the licensed portions of the spectrum used by primary users and the unlicensed portions of the spectrum through wideband access technology. Consequently, the operation types for CR networks can be classified as licensed band operation and unlicensed band operation.

- Licensed band operation: The licensed band is primarily used by the primary network. Hence, CR networks are focused mainly on the detection of primary users in this case. The channel capacity depends on the interference at nearby primary users. Furthermore, if primary users appear in the spectrum band occupied by CR users, CR users should vacate that spectrum band and move to available spectrum immediately.

- Unlicensed band operation: In the absence of primary users, CR users have the same right to access the spectrum. Hence, sophisticated spectrum sharing methods are required for $\mathrm{CR}$ users to compete for the unlicensed band.

\section{Network Heterogeneity}

As shown in Fig. 2, the CR users have the opportunity to perform three different access types:

- CR network access: CR users can access their own CR base station, on both licensed and unlicensed spectrum bands. Because all interactions occur inside the CR network, 


CR networks impose
unique challenges
due to the
coexistence with
primary networks, as
well as diverse QoS
requirements. Thus,
new spectrum
management
functions are
required for $C R$
networks to meet
critical design
challenges.

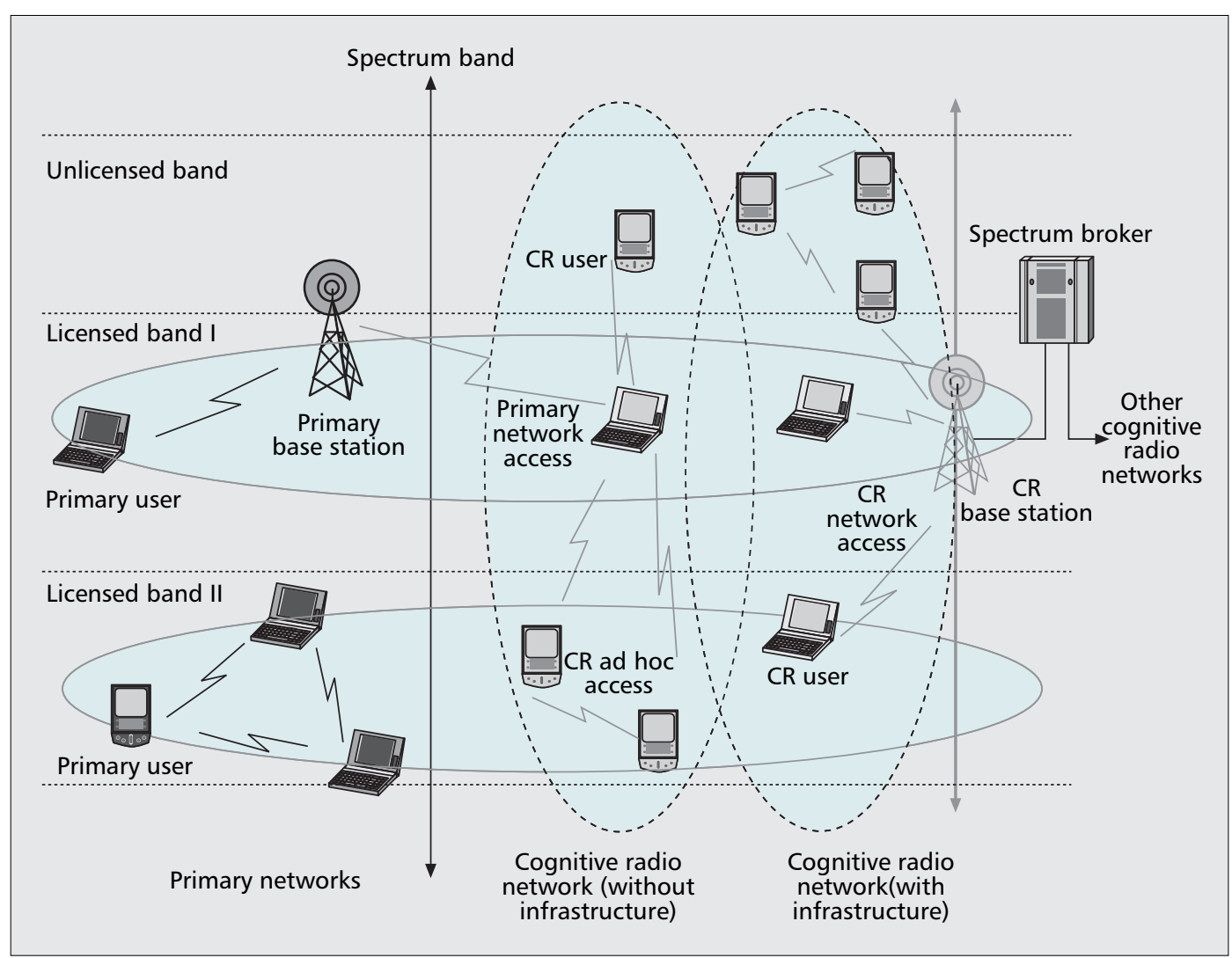

Figure 2. Cognitive radio network architecture.

their spectrum sharing policy can be independent of that of the primary network.

- $C R$ ad hoc access: CR users can communicate with other CR users through an ad hoc connection on both licensed and unlicensed spectrum bands.

- Primary network access: CR users can also access the primary base station through the licensed band. Unlike for other access types, CR users require an adaptive medium access control (MAC) protocol, which enables roaming over multiple primary networks with different access technologies.

According to the CR architecture shown in Fig. 2, various functionalities are required to support spectrum management in CR networks. An overview of the spectrum management framework and its components is provided next.

\section{Spectrum Management Framework}

CR networks impose unique challenges due to their coexistence with primary networks as well as diverse QoS requirements. Thus, new spectrum management functions are required for CR networks with the following critical design challenges:

- Interference avoidance: CR networks should avoid interference with primary networks.

- QoS awareness: To decide on an appropriate spectrum band, CR networks should support QoS-aware communication, considering the dynamic and heterogeneous spectrum environment.

- Seamless communication: CR networks should provide seamless communication regardless of the appearance of primary users.

To address these challenges, we provide a directory for different functionalities required for spectrum management in CR networks. The spectrum management process consists of four major steps:

- Spectrum sensing: A CR user can allocate only an unused portion of the spectrum. Therefore, a CR user should monitor the available spectrum bands, capture their information, and then detect spectrum holes.

- Spectrum decision: Based on the spectrum availability, CR users can allocate a channel. This allocation not only depends on spectrum availability, but is also determined based on internal (and possibly external) policies.

- Spectrum sharing: Because there may be multiple CR users trying to access the spectrum, CR network access should be coordinated to prevent multiple users colliding in overlapping portions of the spectrum.

- Spectrum mobility: CR users are regarded as visitors to the spectrum. Hence, if the specific portion of the spectrum in use is required by a primary user, the communication must be continued in another vacant portion of the spectrum.

The spectrum management framework for $\mathrm{CR}$ network communication is illustrated in Fig. 3 . It is evident from the significant number of interactions that the spectrum management functions require a cross-layer design approach. In the following sections we discuss the four main spectrum management functions. 


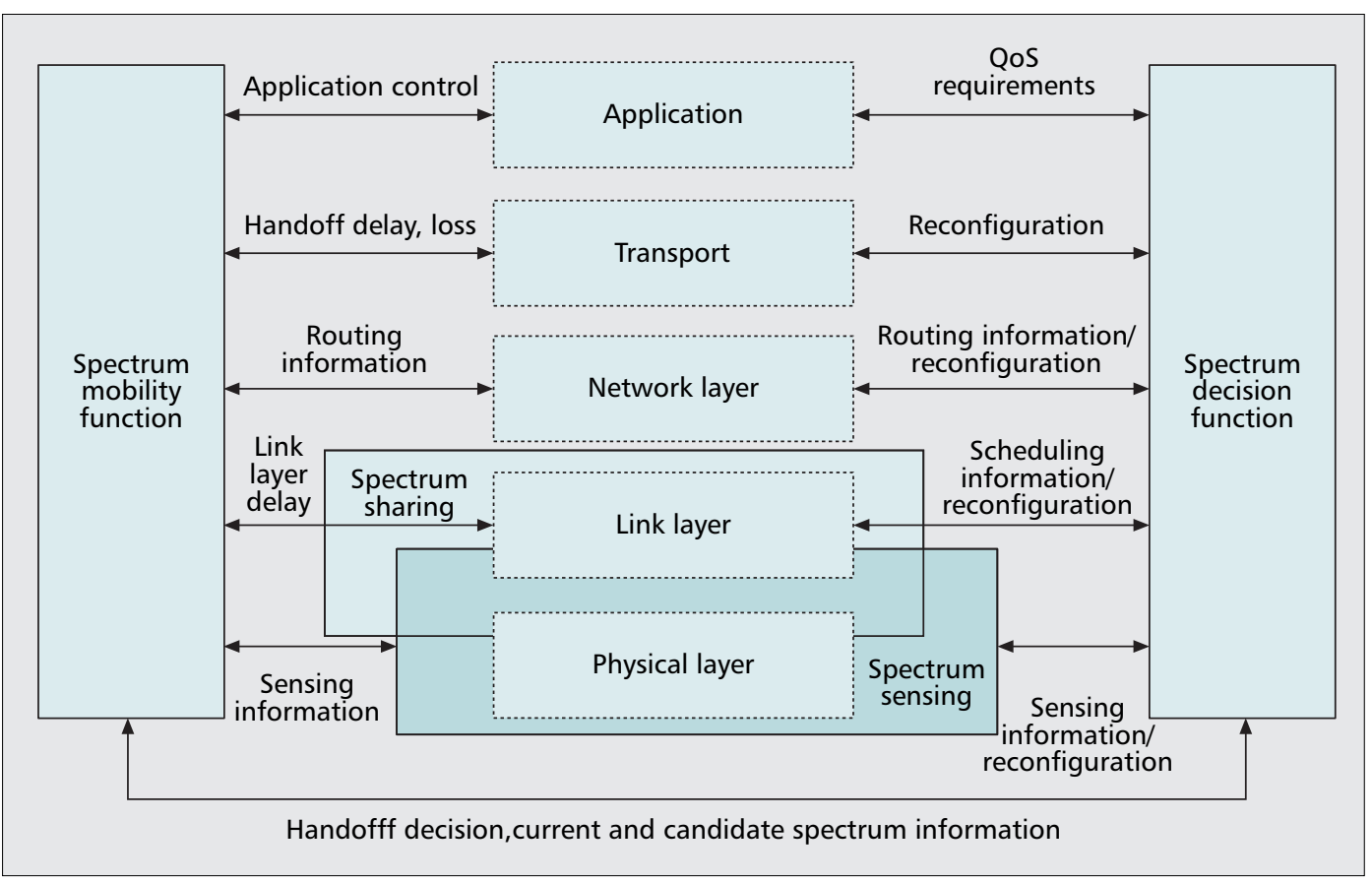

A cognitive radio is

designed to be

aware of and

sensitive to the

changes in its

surroundings, which

makes spectrum

sensing an important

requirement for the

realization of $C R$

networks.

Figure 3. Spectrum management framework for cognitive radio networks.

\section{SPECTRUM SENSING}

A CR is designed to be aware of and sensitive to the changes in its surroundings, which makes spectrum sensing an important requirement for the realization of CR networks. Spectrum sensing enables CR users to adapt to the environment by detecting spectrum holes without causing interference to the primary network. This can be accomplished through a real-time wideband sensing capability to detect weak primary signals in a wide spectrum range. Generally, spectrum sensing techniques can be classified into three groups: primary transmitter detection, primary receiver detection, and interference temperature management as described in the following.

\section{Primary TRansmitter Detection}

Transmitter detection is based on the detection of a weak signal from a primary transmitter through the local observations of CR users. Three schemes are generally used for transmitter detection: matched filter detection, energy detection, and feature detection [5]:

- Matched filter detection: When the information of the primary user signal is known to the CR user, the optimal detector in stationary Gaussian noise is the matched filter. However, the matched filter requires a priori knowledge of the characteristics of the primary user signal.

- Energy detection: If the receiver cannot gather sufficient information about the primary user signal, the optimal detector is an energy detector. However, the performance of the energy detector is susceptible to uncertainty in noise power. Also, energy detectors often generate false alarms triggered by unintended signals because they cannot differentiate signal types.
- Feature detection: In general, modulated signals are characterized by built-in periodicity or cyclostationarity. This feature can be detected by analyzing a spectral correlation function [7]. The main advantage of feature detection is its robustness to uncertainty in noise power. However, it is computationally complex and requires significantly long observation times.

Due to the lack of interactions between primary users and CR users, transmitter detection techniques rely only on weak signals from the primary transmitters. Hence, transmitter detection techniques alone cannot avoid interference to primary receivers because of the lack of primary receiver information as depicted in Fig. 4a. Moreover, transmitter detection models cannot prevent the hidden terminal problem. A CR user (transmitter) can have a good line of sight to a $\mathrm{CR}$ receiver but may not be able to detect the primary transmitter due to shadowing, as shown in Fig. 4b. Therefore, sensing information from other users is required for more accurate primary transmitter detection - referred to as cooperative detection.

Cooperative detection is theoretically more accurate because the uncertainty in a single user's detection can be minimized through collaboration [8]. Moreover, multipath fading and shadowing effects can be mitigated so that the detection probability is improved in a heavily shadowed environment. However, cooperative approaches cause adverse effects on resourceconstrained networks due to the overhead traffic required for cooperation.

\section{Primary Receiver Detection}

Although cooperative detection reduces the probability of interference, the most efficient way to detect spectrum holes is to detect the primary users that are receiving data within the 


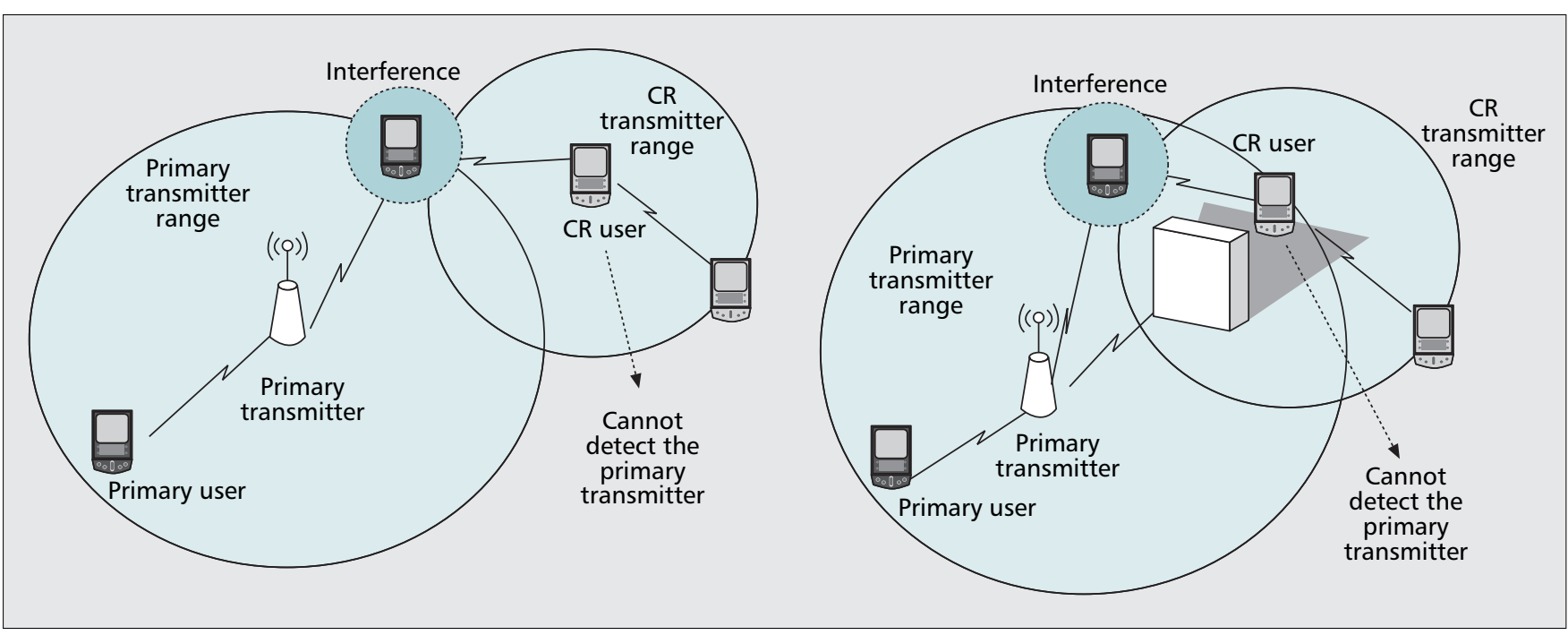

Figure 4. Transmitter detection problem: a) receiver uncertainty; b) shadowing uncertainty.

communication range of a $\mathrm{CR}$ user. Usually, the local oscillator (LO) leakage power emitted by the RF front-end of the primary receiver is exploited [9]. However, because the LO leakage signal is typically weak, implementation of a reliable detector is not trivial. Currently, this method is only feasible in the detection of TV receivers.

\section{INTERFERENCE TEMPERATURE MANAGEMENT}

Traditionally, interference can be controlled at the transmitter through the radiated power and location of individual transmitters. However, interference actually takes place at the receivers, as shown in Fig. 4a. Therefore, recently a new model for measuring interference, referred to as interference temperature, has been introduced by the Federal Communications Commission (FCC) [1]. This model limits the interference at the receiver through an interference temperature limit, which is the amount of new interference the receiver could tolerate. As long as CR users do not exceed this limit, they can use the spectrum band. Although this model is the best fit for the objective of spectrum sensing, the difficulty of this model lies in accurately determining the interference temperature limit.

\section{Spectrum Sensing Challenges}

There exist several open research challenges that must be investigated for the development of spectrum sensing techniques:

- Interference temperature measurement: Due to the lack of interactions between primary networks and CR networks, generally a CR user cannot be aware of the precise locations of the primary receivers. Thus, new techniques are required to measure or estimate the interference temperature at nearby primary receivers.

- Spectrum sensing in multi-user networks: The multi-user environment, consisting of multiple CR users and primary users, makes it more difficult to sense spectrum holes and estimate interference. Hence, spectrum sensing functions should be developed considering the multi-user environment.
- Spectrum-efficient sensing: Sensing cannot be performed while transmitting packets. Hence, CR users should stop transmitting while sensing, which decreases spectrum efficiency. For this reason, balancing spectrum efficiency and sensing accuracy is an important issue. Moreover, because sensing time directly affects transmission performance, novel spectrum sensing algorithms must be developed such that the sensing time is minimized within a given sensing accuracy.

\section{SPECTRUM DeCision}

CR networks require the capability to decide which is the best spectrum band among the available bands according to the QoS requirements of the applications. This notion is called spectrum decision and constitutes a rather important but as yet unexplored topic in CR networks.

Spectrum decision is closely related to the channel characteristics and operations of primary users. Furthermore, spectrum decision is affected by the activities of other CR users in the network. Spectrum decision usually consists of two steps: first, each spectrum band is characterized, based on not only local observations of CR users but also statistical information of primary networks. Then, based on this characterization, the most appropriate spectrum band can be chosen. In the following we investigate the channel characteristics, decision procedures, and research challenges in CR networks.

\section{Channel Characteristics in Cognitive RAdio Networks}

Because available spectrum holes show different characteristics that vary over time, each spectrum hole should be characterized considering both the time-varying radio environment and spectrum parameters, such as operating frequency and bandwidth. Hence, it is essential to define parameters that can represent a particular spectrum band as follows:

- Interference: From the amount of interfer- 


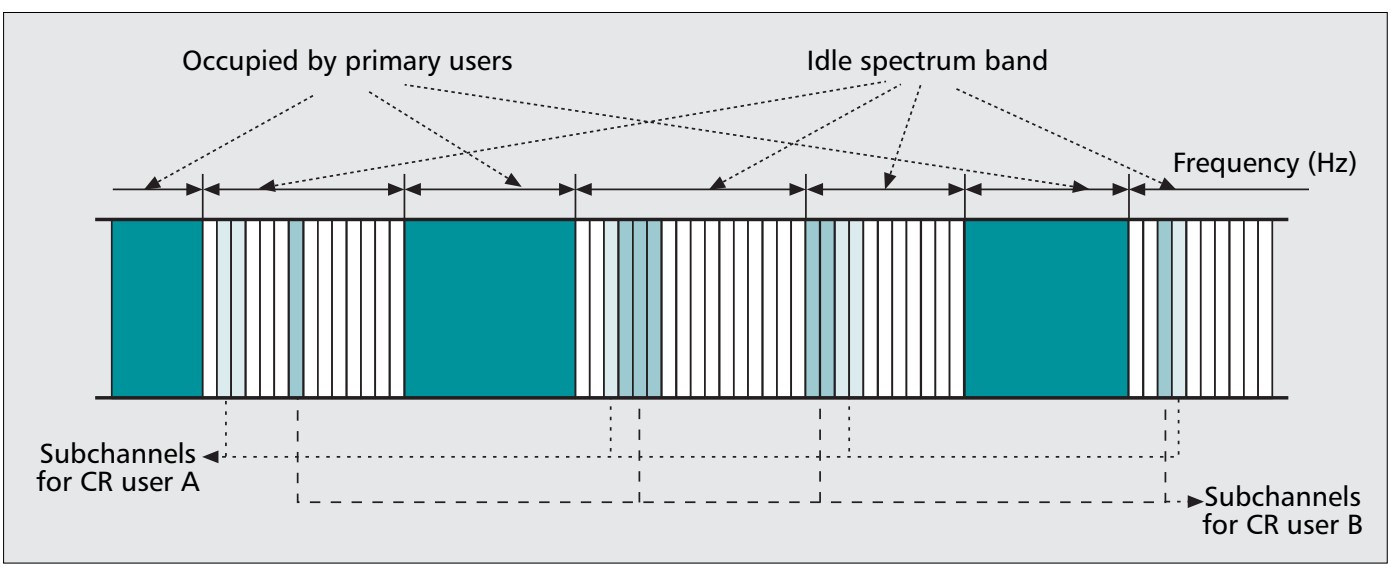

$$
\begin{gathered}
\text { Because the available } \\
\text { spectrum holes show } \\
\text { different characteris- } \\
\text { tics that vary over } \\
\text { time, each spectrum } \\
\text { hole should be } \\
\text { characterized } \\
\text { considering both the } \\
\text { time-varying radio } \\
\text { environment and } \\
\text { the spectrum } \\
\text { parameters, such as } \\
\text { operating frequency } \\
\text { and bandwidth. }
\end{gathered}
$$

ence at the primary receiver, the permissible power of a CR user can be derived, which is used for the estimation of channel capacity.

- Path loss: The path loss is closely related to distance and frequency. As the operating frequency increases, the path loss increases, which results in a decrease in the transmission range. If transmission power is increased to compensate for the increased path loss, interference at other users may increase.

- Wireless link errors: Depending on the modulation scheme and the interference level of the spectrum band, the error rate of the channel changes.

- Link layer delay: To address different path loss, wireless link error, and interference, different types of link layer protocols are required at different spectrum bands. This results in different link layer delays. It is desirable to identify the spectrum bands that combine all the characterization parameters described previously for accurate spectrum decision. However, a complete analysis and modeling of spectrum in CR networks has not been developed yet.

\section{DeCISION PROCEDURE}

After the available spectrum bands are characterized, the most appropriate spectrum band should be selected, considering the QoS requirements and spectrum characteristics. Accordingly, the transmission mode and bandwidth for the transmission can be reconfigured.

To describe the dynamic nature of CR networks, a new metric - primary user activity - is proposed [10], which is defined as the probability of a primary user appearance during CR user transmission. Because there is no guarantee that a spectrum band will be available during the entire communication of a CR user, it is important to consider how often the primary user appears on the spectrum band.

However, because of the operation of primary networks, CR users cannot obtain a reliable communication channel for a long time period. Moreover, CR users may not detect any single spectrum band to meet the user's requirements. Therefore, multiple noncontiguous spectrum bands can be simultaneously used for transmis- sion in CR networks, as shown in Fig. 5. This method can create a signal that is not only capable of high data throughput, but is also immune to interference and primary user activity. Even if spectrum handoff occurs in one of the current spectrum bands, the rest of the spectrum bands will maintain current transmissions.

\section{Spectrum Decision Challenges}

In the development of the spectrum decision function, several challenges still remain unsolved:

- Decision model: Spectrum capacity estimation using signal-to-noise ratio (SNR) is not sufficient to characterize the spectrum band in CR networks. Also, applications require different QoS requirements. Thus, design of application- and spectrum-adaptive spectrum decision models is still an open issue.

- Cooperation with reconfiguration: CR techniques enable transmission parameters to be reconfigured for optimal operation in a certain spectrum band. For example, even if SNR is changed, bit rate and bit error rate (BER) can be maintained by exploiting adaptive modulation instead of spectrum decision. Hence, a cooperative framework with reconfiguration is required in spectrum decision.

- Spectrum decision over heterogeneous spectrum bands: Currently, certain spectrum bands are assigned to different purposes, whereas some bands remain unlicensed. Thus, a CR network should support spectrum decision operations on both the licensed and unlicensed bands.

\section{SPECTRUM SHARING}

The shared nature of the wireless channel requires the coordination of transmission attempts between CR users. In this respect, spectrum sharing should include much of the functionality of a MAC protocol. Moreover, the unique characteristics of CRs, such as the coexistence of CR users with licensed users and the wide range of available spectrum, incur substantially different challenges for spectrum sharing in CR networks. The existing work in spectrum sharing aims to address these challenges and can be classified by four aspects: the architecture, spectrum allocation behavior, spectrum access technique, and scope. 


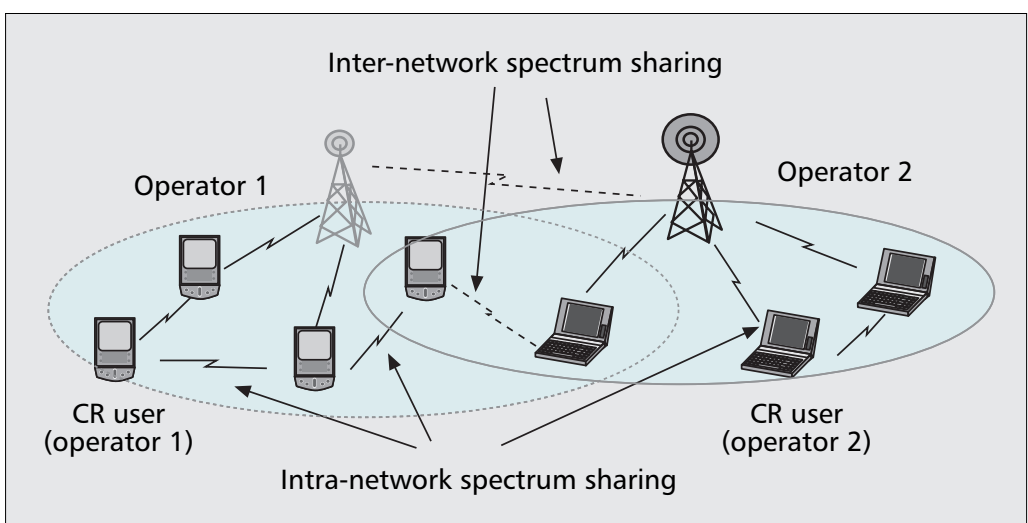

Figure 6. Inter-network and intra-network spectrum sharing in CR networks.

The first classification is based on the architecture, which can be centralized or distributed:

- Centralized spectrum sharing: The spectrum allocation and access procedures are controlled by a central entity. Moreover, a distributed sensing procedure can be used such that measurements of the spectrum allocation are forwarded to the central entity, and a spectrum allocation map is constructed. Furthermore, the central entity can lease spectrum to users in a limited geographical region for a specific amount of time. In addition to competition for the spectrum, competition for users can also be considered through a central spectrum policy server [6].

- Distributed spectrum sharing: Spectrum allocation and access are based on local (or possibly global) policies that are performed by each node distributively [11]. Distributed solutions also are used between different networks such that a base station (BS) competes with its interferer BSs according to the QoS requirements of its users to allocate a portion of the spectrum.

The recent work on comparison of centralized and distributed solutions reveals that distributed solutions generally closely follow the centralized solutions, but at the cost of message exchanges between nodes [12].

The second classification is based on allocation behavior, where spectrum access can be cooperative or noncooperative.

- Cooperative spectrum sharing: Cooperative (or collaborative) solutions exploit the interference measurements of each node such that the effect of the communication of one node on other nodes is considered. A common technique used in these schemes is forming clusters to share interference information locally. This localized operation provides an effective balance between a fully centralized and a distributed scheme.

- Non-cooperative spectrum sharing: Only a single node is considered in non-cooperative (or non-collaborative, selfish) solutions [13]. Because interference in other CR nodes is not considered, non-cooperative solutions may result in reduced spectrum utilization. However, these solutions do not require frequent message exchanges between neighbors as in cooperative solutions.
Cooperative approaches generally outperform noncooperative approaches, as well as closely approximating the global optimum [12]. Moreover, cooperative techniques result in a certain degree of fairness, as well as improved throughput. On the other hand, the performance degradation of non-cooperative approaches are generally offset by the significantly low information exchange and hence, energy consumption.

The third classification for spectrum sharing in CR networks is based on the access technology [14]:

- Overlay spectrum sharing: Nodes access the network using a portion of the spectrum that has not been used by licensed users. This minimizes interference to the primary network.

- Underlay spectrum sharing: The spread spectrum techniques are exploited such that the transmission of a CR node is regarded as noise by licensed users.

Underlay techniques can utilize higher bandwidth at the cost of a slight increase in complexity. Considering this trade-off, hybrid techniques can be considered for the spectrum access technology for CR networks. Finally, spectrum sharing techniques are generally focused on two types of solutions: spectrum sharing inside a CR network (intranetwork spectrum sharing) and among multiple coexisting CR networks (internetwork spectrum sharing), as explained in the following:

- Intranetwork spectrum sharing: These solutions focus on spectrum allocation between the entities of a CR network, as shown in Fig. 6. Accordingly, the users of a CR network try to access the available spectrum without causing interference to the primary users. Intranetwork spectrum sharing poses unique challenges that have not been considered previously in wireless communication systems.

- Internetwork spectrum sharing: The CR architecture enables multiple systems to be deployed in overlapping locations and spectrum, as shown in Fig. 6. So far the internetwork spectrum sharing solutions provide a broader view of the spectrum sharing concept by including certain operator policies.

Next, we describe the potential challenges and open research issues of this aspect.

\section{Spectrum Sharing Challenges}

There are many open research issues for the realization of efficient and seamless open spectrum operation in CR networks, such as:

- Common control channel: A common control channel (CCC) facilitates many spectrum sharing functionalities. However, because a channel must be vacated when a primary user chooses a channel, implementation of a fixed CCC is infeasible. Moreover, in CR networks a channel common to all users is highly dependent on topology and varies over time [15]. Consequently, either $\mathrm{CCC}$ mitigation techniques must be devised or local CCCs must be exploited for clusters of nodes.

- Dynamic radio range: Due to the interdependency between radio range and operating frequency, the neighbors of a node may 
change as the operating frequency changes. So far, there is no work addressing this important challenge in CR networks, and we advocate frequency-aware spectrum sharing techniques.

- Spectrum unit: Almost all spectrum decision and sharing techniques consider a channel as the basic spectrum unit. Hence, the definition of a channel as a spectrum unit is crucial in developing algorithms.

- Location information: An important assumption in the existing work is that secondary users know the location and transmit power of primary users so that interference calculations can be performed easily. However, such an assumption may not always be valid in CR networks.

\section{SPECTRUM MOBILITY}

The fourth step of spectrum management, as explained earlier, is spectrum mobility management. After a CR captures the best available spectrum, primary user activity on the selected spectrum may necessitate that the user change its operating spectrum band(s), which is referred to as spectrum mobility. Spectrum mobility gives rise to a new type of handoff in CR networks, spectrum handoff. Protocols for different layers of the network stack must adapt to the channel parameters of the operating frequency. Moreover, they should be transparent to spectrum handoff and the associated latency.

Each time a CR user changes its frequency of operation, the network protocols may require modifications to the operation parameters. The purpose of the spectrum mobility management in CR networks is to ensure smooth and fast transition leading to minimum performance degradation during a spectrum handoff. An important requirement of mobility management protocols is information about the duration of a spectrum handoff. This information can be provided by the sensing algorithm. After the latency information is available, the ongoing communications can be preserved with only minimum performance degradation.

The intrinsic characteristics of a CR network give rise to two novel concepts: spectrum mobility and spectrum handoff. So far, there is no research effort to address the problems of spectrum handoff. Although the mobility-based handoff mechanisms that have been investigated in cellular networks may lay the groundwork in this area, there are still open research topics to be investigated.

\section{SPeCtrum Mobility Challenges}

The following are the open research issues for efficient spectrum mobility in CR networks:

- Spectrum mobility in the time domain: CR networks adapt to the wireless spectrum based on the available bands. Because these available channels change over time, enabling QoS in this environment is challenging.

- Spectrum mobility in space: The available bands also change as a user moves from one place to another. Hence, continuous allocation of spectrum is a major challenge.

\section{CONCLUSION}

By exploiting the existing wireless spectrum opportunistically, CR networks are being developed to solve current wireless network problems resulting from the limited available spectrum and the inefficiency in spectrum usage. CR networks, equipped with the intrinsic capabilities of cognitive radio, will provide an ultimate spectrum-aware communication paradigm in wireless communications. In this survey intrinsic properties and current research challenges of spectrum management in CR networks are presented. In particular, we investigate novel spectrum management functionalities such as spectrum sensing, spectrum decision, spectrum sharing, and spectrum mobility. Many researchers are currently engaged in developing the communication technologies and protocols required for CR networks. However, to ensure efficient spectrumaware communication, more research is required along the lines introduced in this survey.

\section{REFERENCES}

[1] FCC, ET Docket No 03-322 Notice of Proposed Rule Making and Order, Dec 2003.

[2] I. F. Akyildiz et al., "NeXt Generation/Dynamic Spectrum Access/Cognitive Radio Wireless Networks: A Survey," Comp. Networks J., vol. 50, Sept. 2006, pp. 2127-59.

[3] S. Haykin, "Cognitive Radio: Brain-Empowered Wireless Communications," IEEE JSAC, vol. 23, no. 2, Feb. 2005, pp. 201-20.

[4] F. K. Jondral, "Software-Defined Radio - Basic and Evolution to Cognitive Radio," EURASIP J. Wireless Commun. and Networking, 2005.

[5] D. Cabric, S. M. Mishra, and R. W. Brodersen, "Implementation Issues in Spectrum Sensing for Cognitive Radios," Proc. 38th Asilomar Conf. Sig., Sys. and Comp. 2004, Nov. 2004, pp. 772-76.

[6] O. Ileri, D. Samardzija, and N. B. Mandayam, "Demand Responsive Pricing and Competitive Spectrum Allocation via Spectrum Server," Proc. IEEE DYSPAN 2005 Nov. 2005, pp. 194-202.

[7] M. Oner and F. Jondral, "On the Extraction of the Channel Allocation Information in Spectrum Pooling Systems," IEEE JSAC, vol. 25, no. 3, Apr. 2007, pp. 558-65.

[8] S. M. Mishra, A. Sahai, and R. W. Brodersen, "Cooperative Sensing among Cognitive Radios," Proc. IEEE ICC 2006, vol. 4, June 2006, pp. 1658-63.

[9] B. Wild and K. Ramchandran, "Detecting Primary Receivers for Cognitive Radio Applications," Proc. IEEE DYSPAN 2005, Nov. 2005, pp. 124-30.

[10] S. Krishnamurthy et al., "Control Channel Based MACLayer Configuration, Routing and Situation Awareness for Cognitive Radio Networks," Proc. IEEE MILCOM 2005, Oct. 2005, pp. 455-60.

[11] Q. Zhao et al., "Decentralized Cognitive MAC for Opportunistic Spectrum Access in Ad Hoc Networks: A POMDP Framework," IEEE JSAC, vol. 25, no. 3, Apr. 2007, pp. 589-99.

[12] C. Peng, H. Zheng, and B. Y. Zhao, "Utilization and Fairness in Spectrum Assignment for Opportunistic Spectrum Access," ACM Mobile Networks and Applications (MONET), vol. 11, no. 4, Aug. 2006, pp. 555-76.

[13] H. Zheng and L. Cao, "Device-centric Spectrum Management," Proc. IEEE DySPAN 2005, Nov. 2005, pp. 56-65.

[14] R. Menon, R. M. Buehrer, and J. H. Reed, "Outage Probability Based Comparison of Underlay and Overlay Spectrum Sharing Techniques," Proc. IEEE DySPAN 2005, Nov. 2005, pp. 101-9.

[15] J. Zhao, H. Zheng, and G.-H. Yang, "Distributed Coordination in Dynamic Spectrum Allocation Networks," Proc. IEEE DySPAN 2005, Nov. 2005, pp. 259-68.

\section{BIOGRAPHIES}

IAN F. AKYILDIZ (ian@ece.gatech.edu) received his B.S., M.S., and Ph.D. degrees in computer engineering from the University of Erlangen-Nuernberg, Germany, in 1978, 1981, and 1984, respectively. Currently, he is the Ken Byers Dis-
Many researchers are currently engaged in developing the communication technologies and protocols required for $C R$ networks. However, to ensure efficient spectrumaware communication, more research is required along the lines introduced in this survey. 
tinguished Chair Professor at the School of Electrical and Computer Engineering, Georgia Institute of Technology, Atlanta, and director of the Broadband Wireless Networking Laboratory. His current research interests are in cognitive radio networks, wireless sensor networks, and wireless mesh networks. He is an Editor-in-Chief of Computer Networks Journal (Elsevier), as well as the founding editor-inchief of Ad Hoc Network Journal (Elsevier) and PHYCOM: Physical Communication Journal (Elsevier). He received the Don Federico Santa Maria Medal for his services to the Universidad of Federico Santa Maria in 1986. From 1989 to 1998 he served as a National Lecturer for ACM and received the ACM Outstanding Distinguished Lecturer Award in 1994. He received the 1997 IEEE Leonard G. Abraham Prize Award (IEEE Communications Society) for his paper entitled "Multimedia Group Synchronization Protocols for Integrated Services Architectures," published in IEEE Journal of Selected Areas in Communications in January 1996. He received the 2002 IEEE Harry M. Goode Memorial Award (IEEE Computer Society) with the citation "for significant and pioneering contributions to advanced architectures and protocols for wireless and satellite networking." He received the 2003 IEEE Best Tutorial Award (IEEE Communication Society) for his article entitled "A Survey on Sensor Networks," published in IEEE Communications Magazine, August 2002. He also received the 2003 ACM Sigmobile Outstanding Contribution Award with the citation "for pioneering contributions in the area of mobility and resource management for wireless communication networks." He received the 2004 Georgia Tech Faculty Research Author Award for his "outstanding record of publications of papers between 1999-2003." He also received the 2005 Distinguished Faculty Achievement Award from the School of ECE, Georgia Tech. He has been a Fellow of the Association for Computing Machinery (ACM) since 1996.

WON-YEOL LEE (wylee@ece.gatech.edu) received his B.S. and M.S. degrees from the Department of Electronic Engineering, Yonsei University, Seoul, Korea in 1997 and 1999 respectively. Currently, he is a graduate research assistant in the Broadband Wireless Networking Laboratory, Georgia Institute of Technology, pursuing his Ph.D. degree under the supervision of Prof. Ian F. Akyildiz. His current research interests include cognitive radio networks, next generation wireless networks, and wireless sensor networks. From 1999 to 2004 he was a research engineer at the Network R\&D Center and Wireless Multimedia Service Division at LG Telecom, Seoul, Korea.

MeHMET C. VURAN (mcvuran@ece.gatech.edu) received his B.Sc. degree in electrical and electronics engineering from Bilkent University, Ankara, Turkey, in 2002. He received his M.S. and Ph.D. degrees in electrical and computer engineering from the Broadband Wireless Networking Laboratory, School of Electrical and Computer Engineering, Georgia Institute of Technology, in 2004 and 2007, respectively, under the guidance of Prof. Ian F. Akyildiz. Currently, he is an assistant professor in the Department of Computer Science and Engineering at the University of Nebraska-Lincoln. he serves as an Associate Editor for Computer Networks Journal (Elsevier)and Journal of Sensors (Hindawi). He received the 2007 ECE Graduate Research Assistant Excellence Award from the School of Electrical and Computer Engineering, Georgia Institute of Technology, and the 2006 Researcher of the Year Award from the Broadband Wireless Networking Laboratory, School of Elecrical and Computer Engineering, Georgia Institute of Technology. His current research interests include cognitive radio netowrks, cross-layer design, and correlation-based communication for wireless sensor networks, and deep space communication networks.

SHANTIDEV MOHANTY (shantig@ece.gatech.edu) received his B. Tech. (Hon.) degree from the Indian Institute of Technology, Kharagpur, India, in 2000. He received his M.S. and Ph.D. degrees in electrical engineering from the Georgia Institute of Technology, Atlanta, Georgia, in 2003 and 2005, respectively. He is currently working with Intel Corporation, Portland, Oregon. His current research interests include wireless networks, mobile communications, mobility management, ad-hoc and sensor networks, and crosslayer protocol design. He worked as a summer intern for Bell Labs, Lucent Technologies, Holmdel, New Jersey, during the summers of 2002 and 2003 and for Applied Research, Telcordia Technologies, Piscataway, New Jersey, during the summer of 2004 . From 2000 to 2001 , he worked as a mixed signal design engineer for Texas Instruments, Bangalore, India. 